\title{
Resistance fluctuation spectroscopy
}

\author{
G VENKATARAMAN and $V$ BALAKRISHNAN \\ Reactor Research Centre, Kalpakkam 603102 \\ MS received 13 November 1978
}

The purpose of this note is to draw attention to the potentialities of a new technique, namely, Resistance Fluctuation Spectroscopy (RFS), for probing slow atomic movements in solids, particularly those associated with defect kinetics. The idea was first proposed by one of us (GV) at a seminar three years ago. Subsequently, Celasco et al (1976) independently reported the actual observation of resistance fluctuations in thin films of aluminium, attributable to vacancy effects. These results have now been analysed in depth by Balakrishnan and Bansal (1978) who show that in addition to effects associated with the birth and death of vacancies as postulated earlier by Celasco et al there are additional features arising out of migration (diffusion). However, the basis for this type of spectroscopy in general has not been spelt out so far, a lacuna we now seek to fill.

We start by considering a crystalline solid which, for simplicity, we assume to be monatomic. Assuming that the pseudo-potential concept can be applied to the scattering of electrons by the ions, the electrical resistivity can be expressed by the formula (Ziman 1964; Harrison 1966)

$$
\rho=C N \int_{0}^{2} d x x^{3}|w(q)|^{2} S(q), \quad\left(x=q / k_{F}\right),
$$

where $w(q)$ is the form factor (the matrix element corresponding to scattering from a single ion), $N$ is the number of ions in the crystal and $C$ is a constant related to the atomic volume, Fermi energy, etc. The quantity $S(q)$ is the structure factor, well known in diffraction theory. For an explicit consideration of the effect of dynamics on the resistivity, it is advantageous to go back one step in the derivation of the formula for the resistivity in terms of the structure factor (Baym 1964), and to use the relation

$$
S(q)=\int_{-\infty}^{\infty} d \omega S(q, \omega),
$$

where $S(q, \omega)$ is the dynamic structure factor introduced first by Van Hove (1954). (We are here concerned with the classical limit which is valid for $T \geqslant \theta_{D}$ ). It is useful to note here that

$$
S(q)=S(q, t=0),
$$


where $S(q, t)$ is the Fourier transform of $S(q, \omega)$. The quantity $S(q, t)$ is sometimes called the intermediate scattering function.

The usual contribution to resistivity comes from lattice vibrations, and can be obtained by calculating $S(q, \omega)$ via a phonon expansion. The important point to note here is that the density fluctuations associated with lattice vibrations occur rather rapidly, i.e, in a time $\sim 10^{-12}$ sec. By contrast, the fluctuations associated with defect kinetics (e.g., diffusion) occur at a much slower rate, typically on a time scale $10^{-6} \mathrm{sec}$ or longer. For all practical purposes, therefore, we may write

$$
S(q)=S_{\mathrm{ph}}(q)+\int d \omega S_{\mathrm{D}}(q, \omega),
$$

where $S_{\mathrm{ph}}(q)$ denotes the phonon contribution and $S_{\mathrm{D}}$ that from the slow fluctuations associated with defect kinetics, etc.

In what follows, we shall, for simplicity, assume that the system is in equilibrium, a restriction which can be suitably relaxed if necessary. Confining attention to the slow fluctuations alone, we have a net contribution $\rho_{D}$ obtained by using $\int d \omega S_{D}(q, \omega)$ in place of $S(q)$ in formula (1). This is the contribution when the slow fluctuations are completely averaged over. However, since the fluctuations are slow, the possibility exists of tracking them via 'rapid' observations, whereupon one can draw inferences about the dynamics, as one does while dealing with Brownian motion.

Let $\hat{\rho}_{D}(t)$ denote the instantaneous value of the resistivity, i.e., before averaging over the slow fluctuations. This means $\hat{\rho}_{D}(t)$ will vary in a random fashion; however, it must obviously do so in such a manner that

$$
\left\langle\hat{\rho}_{D}(t)\right\rangle=\rho_{D},
$$

where $\langle>$ implies a suitable average. In terms of the Ziman formula,

$$
\hat{\rho}_{D}(t)=C N \int_{0}^{2} d x x^{3}|w(q)|^{2} \hat{S}_{D}(q, t)
$$

which identifies for us a fluctuating structure factor $\hat{S_{D}}(q, t)$, whose variations are responsible for the variations in $\hat{\rho}_{D}(t)$. Clearly, $\hat{S}_{D}$ must satisfy a relation similar to (5), which is

$$
\left\langle\hat{S}_{D}(q, t)\right\rangle=S_{D}(q)=\int d \omega S_{D}(q, \omega) .
$$

Using modern electronic correlators, one can measure the correlation function $\left\langle\hat{\rho}_{D}(0) \hat{\rho}_{D}(t)\right\rangle$, by suitably converting the resistivity fluctuations into voltage fluctuations. The analysis above shows that dynamics enters through the quantity $\left\langle\hat{S}_{\mathfrak{D}}(q, 0) \hat{S}_{\mathfrak{D}}\left(q^{\prime}, t\right)\right\rangle$. Equivalently, one can also measure the power spectrum

$$
P_{\rho}(\omega)=2 \int_{-\infty}^{\infty} d t\left\langle\hat{\rho}_{D}(0) \hat{\rho}_{D}(t)\right\rangle \exp (i \omega t)
$$

which is what Celasco et al did. However, the simple model used by them for fitting their experimental data, as well as the subsequent theoretical analysis by Balakrishnan and Bansal, were not cast in the structure factor language. The 
main advantage of the latter is the ready link it provides with the standard treatment of atomic dynamics.

A clarification is necessary about the relationship between $S_{D}(q, t)$ introduced in (4) and $\vec{S}_{D}(q, t)$ appearing from (6) onwards. For this purpose, we visualise the system to be so prepared that at an instant of time which we take as $t=0$, the random variable $\hat{S}_{D}(q, t)$ has the numerical value $S_{D}(q)$. Over a period of time $t, \hat{S}_{D}$ will evolve in a random manner, describable perhaps by a suitable stochastic equation. If

$$
P\left[\hat{S}_{D}(q, 1), t \mid S_{D}(q)\right]
$$

denotes the probability that $\hat{S_{D}}$ has the particulat value $\hat{S}_{D}(q .1)$ at time $t$. having started off as above, then

$$
S_{D}(q, t)=\int d \hat{S}_{D}(q, t) P\left[\hat{S}_{D}(q, t), t \mid S_{D}(q)\right] \hat{S_{D}}(q, t)
$$

As an illustration, let us consider a delect undergoing simple diffusion. From earlier work (Vineyard 1958) we know that

$$
S_{D}(q)-1, \quad S_{D}(q, t)=\exp \left(-D q^{2} t\right)
$$

and therefore

$$
\left.S_{D}(\boldsymbol{q}, \omega)=2 D q^{2}\left[\omega^{2}+D_{q}^{\prime}\right)^{p}\right]
$$

where $D$ is tile diffusion coeficient. The power spectum of the fluctuations in $\hat{S}_{D}(q, t)$ would then have the form

$$
\begin{gathered}
4 \int_{0}^{\infty} d t\left\langle\left(S_{D}(q, 0)-\hat{S}_{D}(q)\right)\left(\hat{S}_{D}(q, t)-S_{D}(q)\right\rangle\right. \\
=\quad(\text { const, }) D_{q^{2}} /\left[\omega^{2},\left(D q^{2}\right)^{2}\right] .
\end{gathered}
$$

A random phase approximation with respect to the $q$-dependence (as is standard in the derivation of the usual resistivity formula) is implied in the above. More complicated diffusion dynamics would be reflected very conveniently in the measured power spectrum in an RFS experiment.

It is pertinent to point out that there are certain important differences between RFS and intensity fluctuation spectroscopy (IFS) employed in light scattering (Berne and Pecora 1976), although one probes dynamical correlation effects in both. One difference (aside from the fact that RFS is applicable to metals and IFS to insulators) is that in a light scattering experiment one samples fluctuations at a specific value of $q$ (and a small one at that), whereas in RFS all values of $q$ (upto $2 k_{F}$ ) contribute. More significant is the fact that the phonon 'equilibration' times in the resistivity problem are sufficiently rapid to permit the separation of time scales implied in (4). In turn, this enables us to associate the 'instantaneous ' resistivity $\rho_{D}(t)$ with the corresponding fluctuating structure factor $S_{D}(q, t)$. Such an association is not manifestly permissible in the case of the scattered light intensity, so that IFS must necessarily deal with a fourth order density-density corre- 
lation. Further input information (e.g., the Gaussian approximation, etc.) is then necessary to write this correlation in terms of more easily handled secondorder correlations (Berne and Pecora 1976).

The utility of RFS in practice will undoubtedly depend on the ability to detect and measure the fluctuations involved. However, the preliminary experiments of Celasco et al offer sufficient encouragement. Among the problems to which it can be applied are hydrogen diffusion in metals, martensitic transformations, and the diffusion of kinks on dislocations. The analysis can also be suitably modified to deal with non-equilibrium situations like annealing, creep. irradiation, etc. In the case of creep, for example, RFS camnot only give information over a wide range of frequencies but can also possibly reduce drastically the time required for obtaining information about dynamics. This in turn will facilitate experimentation over a wide range of variation of other parameters such as stress, lemperature, etc.

\section{References}

Balakrishnan V and Bansal N K 1978 (submitted 10 Pramäna)

Baym G 1964 Phys. Rev. 135A 1691

Berne B J and Pecora R 1976 Dinumic light kcutrering (New Yotk: John Wiley)

Celasco M. Fiorillo F and Mazzetif P 1976 Phys. Rev. Lett. 3638

Harrison W A 1966 Pseudopotentials in the theory of metals (Reading, Mass.: Benjamin)

Van Hove L 1954 Plyy. Rev. 95249

Vineyard G H 1958 Phys. Rel. 110999

Ziman J M 1964 Pinciples of the theory of solids (Cambridge : University Press) 